\title{
Eficacia de un ácido kaurénico extraído de la planta venezolana Wedelia trilobata (Asterácea) contra Leishmania (Viannia) braziliensis
}

\author{
Solanny Brito ${ }^{1}$, Oscar Crescente ${ }^{1}$, Alexis Fernández ${ }^{2}$, Aura Coronado ${ }^{1}$, Noris Rodriguez ${ }^{2}$ \\ ${ }^{1}$ Universidad de Oriente, Cumaná, Estado Sucre, Venezuela. \\ ${ }^{2}$ Instituto de Biomedicina, Universidad Central de Venezuela, Caracas, Venezuela.
}

Introducción. La leishmaniasis es un grave problema de salud pública y aun no existe un tratamiento eficaz para la enfermedad. Por esta razón, es necesario investigar nuevas alternativas terapéuticas.

Objetivo. En este trabajo nos propusimos evaluar el efecto parasiticida de un ácido kaurénico (ent-kaur-16-in-19-oico) aislado de la planta superior venezolana denominada Wedelia trilobata (Asteracea) sobre Leishmania (V.) braziliensis in vivo e in vitro.

Materiales y métodos. Los estudios in vitro fueron realizados en amastigotes axénicos, promastigotes así como en macrófagos infectados y no infectados, los cuales fueron tratados con dosis entre $25-100 \mu \mathrm{g} / \mathrm{ml}$ del ácido kaurénico. El efecto del compuesto sobre la viabilidad celular fue evaluado utilizando el método de conteo directo en una cámara de Neubauer o hemocitómetro y por el método indirecto utilizando el método de MTT (Metil Tiazol Tetrazolium). Para el ensayo in vivo, se utilizaron ratones Balb/c infectados subcutáneamente en la almohadilla plantar con $1 \times 10^{6}$ promastigotes de Leishmania (V.) braziliensis; posteriormente los ratones fueron tratados intraperitonealmente durante una semana con $30 \mathrm{mg}$ del ácido kaurénico por $\mathrm{kg}$ de peso, en un volumen de $100 \mu \mathrm{l}$ de etanol al $10 \%$.

Resultados. Este compuesto mostró un potente efecto parasiticida tanto sobre los amastigotes axénicos como sobre promastigotes con dosis letales $50\left(\mathrm{DL}_{50}\right)$ de 0,25 y 0,78 $\mu \mathrm{g} / \mathrm{ml}$ respectivamente, en 24 horas. Se observó una baja toxicidad del compuesto sobre macrófagos de la línea J774G8, con una $\mathrm{DL}_{50}$ de $25 \mu \mathrm{g} / \mathrm{ml}$, manteniéndose una alta viabilidad $(70-92 \%)$ de los mismos, y una moderada viabilidad para los macrófagos infectados (37-81\%) con concentraciones menores de $25 \mu \mathrm{g} / \mathrm{ml}$. Adicionalmente, se observó una clara reducción (70\%) en el tamaño de las lesiones de los ratones tratados sin efectos tóxicos aparentes.

Conclusión. Los resultados obtenidos indican que este compuesto posee un potente efecto leishmanicida sobre Leishmania (Viannia) braziliensis.

Palabras clave: Leishmania, leishmaniasis, quimioterapia.

Efficacy of a kaurenic acid extracted from the Venezuelan plant Wedelia trilobata(Asteracea) against Leishmania (Viannia) braziliensis

Introduction. Leishmaniasis is a global public health problem without adequate treatment options, hence requiring research on new drug development.

Objective. Our main objective was the evaluation of a kaurenic acid (ent-kaur-16-in-19-oico), isolated from the Venezuelan plant Wedelia trilobata (Asteracea), on Leishmania (V) braziliensis both in vivo and in vitro.

Materials and methods. The in vitro assay was performed using axenic amastigotes and promastigotes as well as infected and uninfected macrophages. Parasites and macrophages were treated with concentrations of the compound varying between 10 and $250 \mu \mathrm{g} / \mathrm{ml}$. The effect of the compound on cellular viability was evaluated by counting dead and living cells in a hemocytometer and by the colorimetric method using MTT (Methylthiazoletetrazolium). For the in vivo assay, Balb/c mice were infected subcutaneously with $1 \times 10^{6}$ promastigotes of L.(V.) braziliensis and afterwards treated with a daily dose of $30 \mathrm{mg} / \mathrm{kg}$ in $100 \mu \mathrm{l}$ of kaurenic acid administered by intraperitoneal injection for one week. 
Results. The compound had a lethal effect on axenic amastigotes and promastigotes with $\mathrm{DL}_{50}$ of 0.25 and $0.78 \mu \mathrm{g} / \mathrm{ml}$, respectively, in 24 hours. Low toxicity was observed for J774-G8 macrophages with a $\mathrm{DL}_{50}$ of $25 \mu \mathrm{g} / \mathrm{ml}$ and high viability $(70-92 \%)$, while a moderate viability was observed for infected macrophages (37-81\%), with concentrations of $25 \mu \mathrm{g} / \mathrm{ml}$ or less. Additionally, a $70 \%$ reduction was observed in the size of the skin lesions in Balb/c mice with no evident toxic effect.

Conclusion. The results indicate that this compound has a potent leishmanicidal effect on $L$.(V.) braziliensis.

Key words: Leishmania, leishmaniasis, drug therapy.

La leishmaniasis se distribuye en las zonas tropicales y subtropicales de 88 países de Europa, África, Asia y América, de los cuales 22, países pertenecen a América Latina (1). Esta enfermedad constituye un serio problema de salud pública ocupando el sexto lugar en importancia dentro de las enfermedades tropicales, debido a que produce mutilaciones, deformaciones e incapacidades.

Por más de 70 años, el tratamiento de elección contra la leishmaniasis se ha basado en la quimioterapia convencional con antimoniales pentavalentes como el estibogluconato de sodio (Pentostan $\AA$ ) y el antimoniato de meglumina (Glucantime ${ }^{\circledR}$ ). El uso de estas drogas contra la leishmaniasis se ha asociado con la aparición de reacciones adversas de tipo cardiovascular, renal, hepática y gastrointestinal; a esto se le adicionan las dificultades para su administración por largos periodos de tratamiento, el desconocimiento de su mecanismo de acción, los elevados costos y la resistencia que han desarrollado los parásitos a estos medicamentos, lo cual limita su uso en el tratamiento contra la leishmaniasis (2-5).

En la búsqueda de tratamientos alternativos para los enfermos de leishmaniasis, se han utilizado algunas otras drogas comerciales tales como pentamidina, anfotericina $B$, alopurinol, azoles, ketoconazol, sulfonas, metronidazol y otras, las cuales han resultado tóxicas y poco efectivas, sobre todo, en casos de leishmaniasis difusa y visceral (6-8). Recientemente, una nueva droga denominada miltefosina ha sido utilizada con éxito

\section{Correspondencia:}

Noris Rodríguez, apartado postal 4043, Caracas 1010A, Venezuela

Telefax: (0058) (212) 8648624

nrodri@movistar.net.ve

Recibido: 12/07/05; aceptado: 10/02/06 en el tratamiento de la leishmaniasis visceral en India; sin embargo, se ha reportado resistencia por parte de parásitos de la especie Leishmania donovani a este compuesto $(9,10)$.

Los modelos experimentales, así como los cultivos in vitro de promastigotes, se están utilizando para evaluar algunos compuestos de valor potencial para el tratamiento contra la leishmaniasis. En este sentido, el efecto de un derivado de bifosfonato (pamidronato), compuesto utilizado en el tratamiento de la osteoporosis, ha sido evaluado contra la leishmaniasis experimental, y ha mostrado un total efecto parasiticida con la consecuente desaparición de las lesiones (11).

Más recientemente, en Venezuela se ha comenzado a aislar productos naturales de plantas superiores entre las que se encuentra Wedelia trilobata (Asterácea), la cual biosintetiza un gran número de metabolitos secundarios llamados kaurenos (12), los cuales son diterpenos que contienen un rígido esqueleto tetracíclico (13). Los kaurenos son intermediarios en la biosíntesis de numerosos metabolitos de plantas y hongos, como la giberelina, que poseen propiedades esterodoigénicas, anticonceptivas, hipotensivas, antiinflamatorias, antibióticas, antiparasiticas y citotóxicas (14).

Analizando extractos crudos de W. trilobata, se han logrado caracterizar diversos tipos de diterpenos, entre los cuales se encuentra el ácido kaurénico (ent-kaur-16-en-19-oico) el cual presenta la fórmula molecular $\mathrm{C}_{20} \mathrm{H}_{30} \mathrm{O}_{2}$ (13), y se ha demostrado que este compuesto posee diversas actividades biológicas, entre las cuales se encuentran, la actividad contra Staphylococus aureus y Bacillus cereus, lo cual también fue demostrado con el mismo ácido aislado de Copifera paupera y plantas del género Espeletia, 
$\mathrm{y}$, además, una potente actividad sobre Mycobacterium smegmatis y células tumorales $(15,16)$.

En este trabajo nos propusimos evaluar el efecto del ácido kaurénico (ent-kaur-16-en-19-oico) extraído de W. trilobata sobre Leishmania (Viannia) braziliensis, el agente causal del $80 \%$ de los casos de la leishmaniasis cutánea localizada en Venezuela, en la búsqueda de una quimioterapia más racional contra la leishmaniasis.

\section{Materiales y métodos}

\section{Preparación del material de origen vegetal}

El ácido kaurénico utilizado en este trabajo fue obtenido en el Laboratorio de Productos Naturales, Departamento de Química, Núcleo Sucre, Universidad de Oriente, Cumaná, Venezuela.

El procedimiento utilizado para la obtención de este compuesto es el siguiente: se utilizan los tallos de la planta $W$. trilobata, se secan y se pulverizan; el material pulverizado se macera en solventes orgánicos de diferentes polaridades, primero, hexano y, luego, etanol en una proporción 2:1; después de 24 horas de incubación, el macerado se filtra al vacío y se evapora el solvente para concentrar la muestra. El extracto seco se disuelve en hexano y se preabsorbe en sílica-gel; luego, se coloca en una columna del mismo material; la elución de los productos se inicia con $100 \%$ de hexano, el cual se va variando de acuerdo con la polaridad. Los productos eluidos se analizan por cromatografía en capa fina sobre sílica-gel 60 , bajo luz UV. La determinación de los componentes activos se realiza mediante técnicas espectros-cópicas de resonancia magnética de protones y de carbono $13\left({ }^{1} \mathrm{H}\right.$ y $\left.{ }^{13} \mathrm{C}\right)$, además de espectro infrarrojo y espectrofotometría de masas (13). Se disuelven $50 \mathrm{mg}$ de ácido kaurénico en metanol al $10 \%$ para los ensayos in vitro; para los ensayos in vivo se usó etanol al $10 \%$ como solvente, por encontrarse que este alcohol es menos irritante para los ratones.

\section{Cultivo y mantenimiento de parásitos y macrófagos}

Se mantuvieron promastigotes de L.(V.) braziliensis (MHOM/BR/84/LTB300) y macrófagos de la línea tumoral J774-G8 en medio SDM-79
(17) y RPMI-1640 (JRH-Bioscience y Gibco-BRL), respectivamente, con suplemento de $10 \%$ de suero fetal bovino (SFB); para el cultivo de parásitos, el SFB se inactivó a $56^{\circ} \mathrm{C}$ durante 30 minutos.

Se recolectaron los promastigotes en la fase logarítmica tardía de crecimiento por centrifugación a 3.000 rpm, se lavaron tres veces en solución salina amortiguada con fosfatos (PBS, $\mathrm{pH} 8,0) \mathrm{y}$, finalmente, se resuspendieron en medio fresco y se ajustaron a una concentración de $1 \times 10^{6} / \mathrm{ml}$. Los macrófagos se mantuvieron en medio RPMI 1640 con $10 \%$ de suero fetal bovino en estufa a $37^{\circ} \mathrm{C}$ con $5 \%$ de $\mathrm{CO}_{2}$. Luego, se recolectaron utilizando un policía de goma, centrifugado a $3.000 \mathrm{rpm}$ y ajustado a una concentración de $1 \times 10^{6} / \mathrm{ml}$.

\section{Ensayos anti-Leishmania in vitro}

Se disolvieron $50 \mathrm{mg}$ de ácido kaurénico en metanol al $10 \%$ y se utilizó en concentraciones entre 10 y $500 \mu \mathrm{g}$ por $10^{6}$ células, incluyendo promastigotes de $L$. $(V$.) braziliensis, macrófagos de la línea J774-G8 infectados y no infectados y amastigotes axénicos. Estos últimos se obtuvieron mediante la transformación de promastigotes en medio RPMI-1640 ( $\mathrm{pH} \mathrm{5,0)} \mathrm{y}$ temperatura de $37^{\circ} \mathrm{C}$ durante 48 horas (18).

Para la obtención de macrófagos infectados, se utilizaron monocapas de macrófagos los cuales fueron infectados con promastigotes de Leishmania en una relación $10: 1$ e incubados a $35^{\circ} \mathrm{C}(19)$; después de 24 horas se descartaban los promastigotes no enlazados. A las 72 horas se contaban los promastigotes dentro de la célula; una vez establecida la infección (5 días), se utilizaron las células infectadas. De la misma forma, los macrófagos no infectados se incubaron durante 5 días; a ambos tipos de células se les cambiaba el medio de cultivo cada 48 horas.

Para cada ensayo, las células se resuspendieron en $100 \mu \mathrm{l}$ de medio SDM-79 $+10 \%$ de SFB (GIBCO), y se colocaron en placas de ELISA de 96 pozos. Para el cálculo de la dosis letal $\left(\mathrm{DL}_{50}\right)$ se utilizó el método de conteo directo al microscopio después de colorear con azul de tripano (vol/vol) para medir la viabilidad, y por el 
método colorimétrico (20), para lo cual los parásitos con las distintas concentraciones de la droga, se incubaron durante 18-24 horas; luego, se adicionaron $10 \mu \mathrm{l}(10 \mathrm{mg} / \mathrm{ml})$ de metil-tiazol tetrazolium (MTT, Sigma), se incubaron por 4 horas y se detuvo la reacción con solución amortiguada de lisis (50\% isopropanol, $10 \%$ SDS), se sometió a agitación durante 10 minutos y, luego, se midió la densidad óptica (DO) a $570 \mathrm{~nm}$. La viabilidad celular es directamente proporcional a la DO puesto que a mayor número de células vivas, mayor intensidad de color, ya que las células vivas tienen mayor capacidad para metabolizar el MTT, trasformándolo en cristales de formazán que son los que generan el color.

Para cada experimento se realizaron los respectivos controles, incluyendo células tratadas con metanol solo, un control sin droga y un control con antimoniato de meglumina.

\section{Ensayos anti-Leishmania in vivo}

Para los ensayos in vivo, se utilizaron 10 ratones $\mathrm{Balb} / \mathrm{c}$, hembras, de 8 semanas de edad con un peso promedio de $26 \mathrm{~g}$, suministrados por el Bioterio del Instituto de Biomedicina.

Los ratones fueron previamente inoculados en la almohadilla plantar con $1 \times 10^{6}$ promastigotes en fase estacionaria de crecimiento; 4 semanas después de la inoculación, cada ratón fue tratado con una inyección diaria $(30 \mathrm{mg} / \mathrm{kg})$ del ácido kaurénico, por vía intraperitoneal. El tratamiento se aplicó durante 7 días, utilizando como control 10 ratones infectados y tratados con etanol al $10 \%$. El seguimiento de la evolución del tratamiento en los ratones se hizo diariamente por medición de las lesiones en la almohadilla plantar, utilizando para ello un calibrador de papel digital (Marathon Instruments).

\section{Aspectos éticos}

Los animales experimentales fueron tratados bajo las normas y principios de la guía internacional de principios para la investigación biomédica con animales de experimentación (21-22).

Así mismo, se obtuvo la correspondiente autorización del Comité de Bioética de nuestra institución.

\section{Resultados}

\section{Obtención de la dosis letal 50 y efecto in vitro}

Los resultados indican que el ácido kaurénico tiene un potente efecto leishmanicida sobre promastigotes, amastigotes axénicos y macrófagos (cuadro 1), en el que podemos observar que, a pesar de que se usa el mismo número de células, la dosis letal $50\left(\mathrm{DL}_{50}\right)$ es diferente para cada tipo celular, y que la menor fue la obtenida para los amastigotes axénicos. Los valores de densidad óptica permiten calcular la $\mathrm{DL}_{50}$, aplicando el algoritmo matemático previamente descrito (20), obteniéndose un valor de $0,25 \mu \mathrm{g} / \mathrm{ml}$ para amastigotes axénicos y 0,80 $\mu \mathrm{g} / \mathrm{ml}$ para promastigotes a las 24 horas de incubación y de $25 \mu \mathrm{g} / \mathrm{ml}$ para macrófagos.

En la figura 1 se muestra el efecto de distintas concentraciones del ácido kaurénico sobre promastigotes de L.(V) braziliensis, observándose que las concentraciones mayores de $25 \mu \mathrm{g} / \mathrm{ml}$ producen la muerte celular. Se observa crecimiento normal de los promastigotes en presencia del metanol utilizado como solvente del ácido kaurénico; el mismo comportamiento se observó en presencia de Glucantime ${ }^{\circledR}$ a concentraciones hasta de $500 \mu \mathrm{g} / \mathrm{ml}$ (no se muestra el resultado).

La figura 2 muestra el efecto del ácido kaurénico sobre macrófagos previamente infectados con promastigotes de L.(V.) braziliensis. Los resultados muestran entre $81,03 \%$ y $37,02 \%$ de viabilidad a una concentración de 12,5 y $25 \mathrm{ug} /$ $\mathrm{ml}$ respectivamente, con respecto al $100 \%$ de viabilidad de los macrófagos sin droga. Estos porcentajes son similares tanto por el método de conteo directo con azul de tripano como por el

Cuadro 1. Dosis letal 50 calculada para promastigotes, amastigotes axénicos y macrófagos J774-G-8.

\begin{tabular}{|c|c|c|c|}
\hline Células x $10^{6}$ & $\begin{array}{l}\mathrm{DO}_{1} \\
\text { a } 10 \mathrm{ug}\end{array}$ & $\begin{array}{c}\mathrm{DO}_{2} \\
\text { a } 500 \mathrm{ug}\end{array}$ & $\begin{array}{c}\mathrm{DL}_{50} \\
(\mu \mathrm{g} / \mathrm{ml})\end{array}$ \\
\hline Promastigotes & 1,542 & 0,048 & $0,80 \pm 0,2$ \\
\hline $\begin{array}{l}\text { Amastigotes } \\
\text { axénicos }\end{array}$ & 0,772 & 0,029 & $0,25 \pm 0,08$ \\
\hline $\begin{array}{l}\text { Macrófagos } \\
\text { J774-G8 }\end{array}$ & 0,828 & 0,0912 & $25 \pm 6,02$ \\
\hline
\end{tabular}




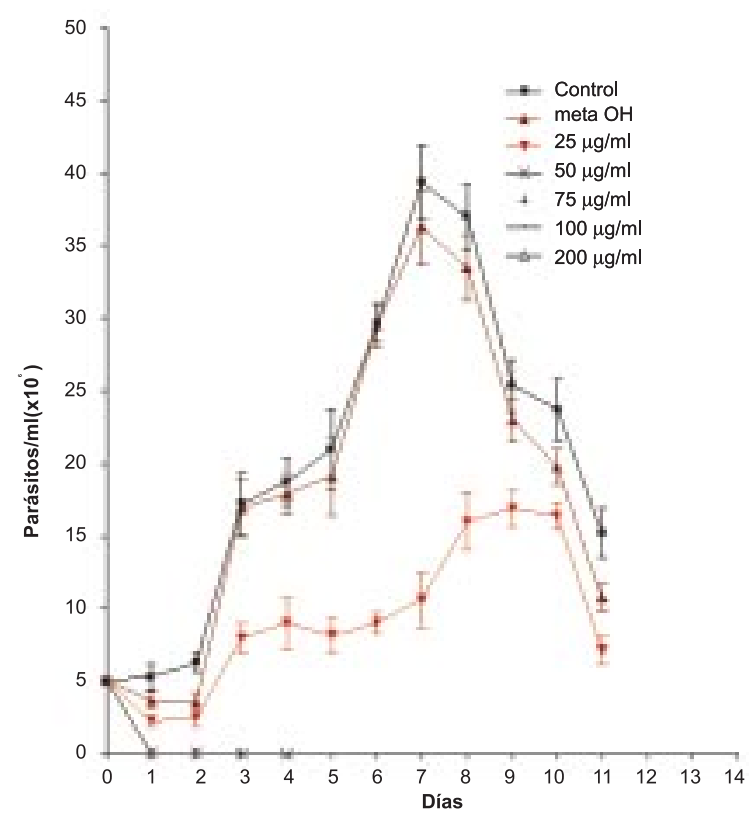

Figura 1. Efecto del ácido kaurénico sobre promastigotes de Leishmania (Viannia) braziliensis, cultivados en medio SDM- $79+10 \%$ de suero fetal bovino y tratados con diversas concentraciones del compuesto.

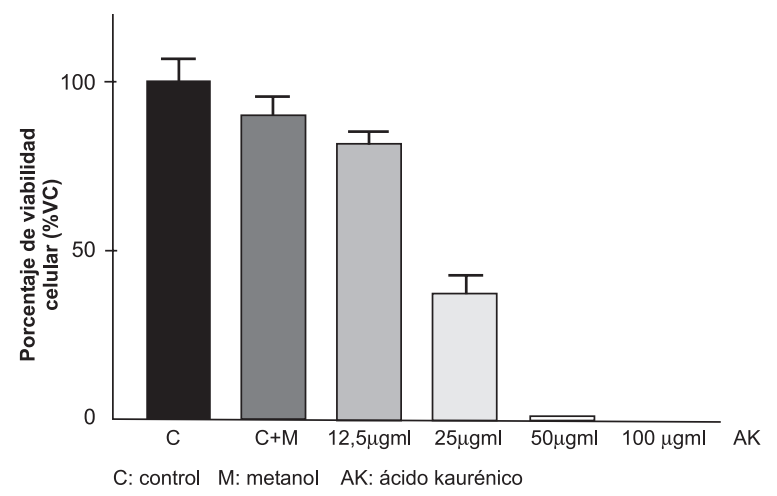

Figura 2. Efecto del ácido kaurénico sobre la línea celular de macrófagos J774-G8 previamente infectados con promastigotes de Leishmania (V) braziliensis. Los macrófagos se infectaron en una relación parásito/ macrófagos 10:1 y, posteriormente, fueron tratados con distintas concentraciones del compuesto.

método indirecto con MTT, lo cual concuerda con la sensibilidad mostrada por los amastigotes axénicos.

\section{Efecto sobre la leishmaniasis experimental}

Se evaluó el efecto del ácido kaurénico extraído de $W$. trilobata sobre la leishmaniasis experimental

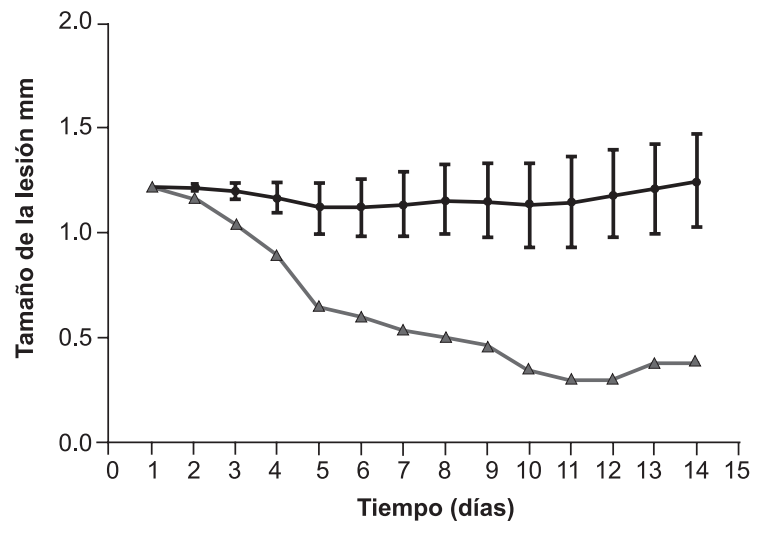

Figura 3. Efecto del ácido kaurénico in vivo. Diez ratones Balb/c por grupo fueron infectados con $1 \times 10^{6}$ promastigotes. Se realizaron medidas diarias del tamaño de la lesión a partir de la aplicación de la primera dosis de ácido kaurénico (30 $\mathrm{mg} / \mathrm{kg}$ de peso).

u Ratones tratados; • Ratones controles.

producida por L. (V.) braziliensis, inducida en ratones Balb/c. La figura 3 muestra los resultados obtenidos después de las mediciones realizadas en la almohadilla plantar de los ratones; las medidas graficadas son el promedio de 10 ratones por grupo, evaluados hasta el día 14 después de la aplicación de 7 dosis (una dosis diaria) de 30 mg de ácido kaurénico por kilogramo de peso; el grupo control fue tratado con etanol $(10 \mu \mathrm{l}$ de etanol en $90 \mu$ de agua).

Se observa una reducción en el tamaño de la lesión, la cual se inicia a partir del primer día de tratamiento, obteniéndose una reducción total de $1,2 \mathrm{~mm}$ lo que representa el $70 \%$ a los 7 días después del tratamiento; esta reducción también se manifiesta en el número de parásitos intracelulares observados en los frotis de las lesiones, teñidos con Giemsa, en los cuales se observaron $5 \pm 3$ parásitos en 10 campos, en comparación con $35 \pm 5$ parásitos en 10 campos en los ratones controles.

\section{Discusión}

El efecto leishmanicida del ácido kaurénico, (entkaur-16-en-19-oico) fue evaluado in vivo e in vitro. Los resultados obtenidos en promastigotes, amastigotes axénicos y macrófagos infectados y sin infectar, utilizando el método colorimétrico para determinar la viabilidad celular $y$, por ende, la $\mathrm{DL}_{50}$ 
(20), indican que este compuesto tiene un alto poder leishmanicida. Se evaluaron diferentes concentraciones de ácido kaurénico, y se encontró una $\mathrm{DL}_{50}$ para promastigotes de $0,80 \mu \mathrm{g} / \mathrm{ml}$ a las 24 horas de incubación con concentraciones entre 10 y $200 \mu \mathrm{g} / \mathrm{ml}$; dosis mayores de $25 \mu \mathrm{g} / \mathrm{ml}$ producen el $100 \%$ de muerte de los promastigotes de L. (V.) braziliensis, dosis menor que la reportada para promastigotes de Leishmania (Leishmania) mexicana (13).

En amastigotes axénicos, la dosis necesaria para eliminar el $50 \%$ de los parásitos fue de $0,25 \mu \mathrm{g} / \mathrm{ml}$ en 24 horas, observándose que para la forma amastigote la $\mathrm{DL}_{50}$ es, aproximadamente, una tercera parte de la dosis necesaria para los promastigotes, lo que indica una mayor susceptibilidad de la forma amastigote, tal como ha sido reportado para el Glucantime® (23), en la que los amastigotes axénicos de $L$. (L.) donovani son susceptibles a una $\mathrm{DL}_{50}$ de $80 \mu \mathrm{g} / \mathrm{ml}$, mientras los promastigotes son susceptibles a una $\mathrm{DL}_{50}$ de $5.830 \mu \mathrm{g} / \mathrm{ml}$ de Glucantime®.

Los resultados aquí reportados, también, sugieren que el ácido kaurénico podría ser estadoespecífico, ya que la $\mathrm{DL}_{50}$ para los amastigotes axénicos es 3,2 veces menor que para los promastigotes, aunque en comparación con Glucantime® tiene un potente efecto leishmanicida sobre ambas formas del parásito.

Se han sintetizado otros compuestos con actividad leishmanicida y se han evaluado in vivo e in vitro; entre ellos se encuentran algunos derivados de la pirimidina y una serie de diarilheptanoides, los cuales han mostrado ser efectivos en promastigotes de $L$. (L.) donovani, L. (L.) amazonensis, L. (V.) braziliensis y L. (L.) chagasi $(24,25)$.

Actualmente, en la búsqueda de mejores medicamentos con menos efectos colaterales para el tratamiento de la leishmaniasis se han explorado los productos naturales derivados de plantas superiores, entre los cuales se encuentran el extracto alcaloide aislado de la planta Aspidosperma ramiflorum (Apocinácea) la cual inhibe el crecimiento de promastigotes de $L$. (L.) amazonensis y L. (V.) braziliensis (26). Así mismo, se ha aislado una serie de compuestos con poder leishmanicida, entre los cuales se encuentran quinonas, lactosas, acetógenos, lignanos, flavonoides y terpenos.

En cuanto a los terpenos, los cuales muestran una gran actividad leishmanicida, son los metabolitos secundarios más abundantes en la naturaleza, ya que juegan un papel muy importante en la biosíntesis de las vitaminas $A, D$ y $E$, de algunas hormonas como la cortisona, el estrógeno y la testosterona; además, intervienen en la síntesis de algunos esteroides, como el colesterol, y forman parte de aceites esenciales y carotenoides. Los diterpenos 7-hidroxi-12-metoxinor-abieta-1,5 (10), 7, 9,12-penta-en-6,14-dione y el abieta-8,12-dien-11,14-dione (12-deoxiroileanone), así como un triterpeno aislado de la raíz de Salvia silicica, mostraron actividad leishmanicida contra amastigotes de $L$. ( $L$ ) donovani y $L$. (L) major a dosis letales de 170 y $290 \mathrm{nM}$, respectivamente (27-29).

Todos estos resultados apoyan la búsqueda de compuestos leishmanicidas derivados de plantas. Nuestro trabajo aporta resultados en cuanto al efecto de estos productos sobre el promastigote, la célula hospedadora y la forma intracelular de Leishmania spp. Los resultados obtenidos utilizando la línea celular J774-G8 indican que a una concentración de $25 \mu \mathrm{g} / \mathrm{ml}$, la viabilidad de los macrófagos es de 70,08\%. Esta concentración es similar a la encontrada para otros derivados de plantas tales como las saponinas en fibroblastos humanos (30); sin embargo, estos valores resultan altos comparados con el efecto del ácido kaurénico en líneas cancerígenas donde se encontró la $\mathrm{DL}_{50}$ entre 0,59 y $0,012 \mu \mathrm{g} / \mathrm{ml}$ (16).

En cuanto al tratamiento experimental de la leishmaniasis cutánea, se pudo observar una reducción de $70 \%$ en el tamaño de la lesión, utilizando una dosis diaria de $30 \mu \mathrm{g} / \mathrm{kg}$ de peso durante 7 días. Sin embargo, no hubo eliminación total de los parásitos en las lesiones, ya que se observaron algunas formas amastigotes (5 en 100 células). Además, el error estándar calculado para los ratones tratados, utilizando el programa PRISMA, no detectó errores significativos en los valores obtenidos, en contraste con los controles cuyos errores estándar fueron variables durante el tiempo de la medición. Este resultado sugiere un 
importante efecto parasiticida in vivo. Es probable que al incrementar la concentración del compuesto o el tiempo de tratamiento, ocurra la eliminación total de los parásitos, tal como ocurre con otros compuestos como el pamidronato, el cual induce una total cura parasitológica en ratones Balb/c (11).

Los resultados sugieren que el ácido kaurénico (ent-kaur-16-en-19-oico-9) aquí reportado sería un posible candidato a ser evaluado en futuras investigaciones utilizando adicionalmente otras vías de inoculación, para valorar su utilidad en el tratamiento de la leishmaniasis cutánea producida por L. (V.) braziliensis.

\section{Agradecimientos}

Al personal del Laboratorio de Ingeniería Genética, por el apoyo prestado durante la realización del trabajo, especialmente a Miguel A. Barrios, por la ayuda prestada en la estandarización del método indirecto para el cálculo de la $\mathrm{DL}_{50}$.

\section{Conflicto de interés}

Los autores declaran no tener conflicto de intereses en el manuscrito.

\section{Financiación}

Este estudio fue realizado con el apoyo económico del CDCH de la Universidad Central de Venezuela y el FONACIT proyecto S1-2001-000761

\section{Referencias}

1. Organización Mundial de la Salud. Special Program for Research and Training in Tropical Disease (TDR). Life in the $21^{\text {st }}$ century. A vision for all. Report of the Director. Geneva: OMS; 1988. p.44-51.

2. Rangel HR, Dagger F, Compagnone RS. Antiproliferative effect of illimaquinone on Leishmania mexicana. Cell Biol Int 1997:21:337-9.

3. Chassaigne JA. Farmacología clínica del antimoniato de meglumina (Glucantime) en Leishmaniasis visceral. Ciencias de la Salud 1998;1:10-5.

4. Loiseau PM, Mbongo N, Bories C, Boulard Y, Craciunesco DG. In vitro antileishmanial action of IR(COD)-pentamidine tetraphenylborate in Leishmania donovani and Leishmania major mouse models. Parasite 2000;7:103-8.

5. Yépez J, Scorza D. Quimioterapia de la leishmaniasis cutánea localizada. Bol Malariol San Amb 2003;43:920.
6. Hellier I, Dereure O, Tournillac I, Pratlong F, Guillot B, Dedet JP et al. Treatment of Old World cutaneous leishmaniasis by pentamidine isothionate. Dermatology 2000;200:120-3.

7. Barata LE, Santos LS, Ferri PH, Phillipson JD, Paine A, Croft SL. Anti-leishmanial activity of neolignans from Virola species and synthetic analogues. Phytochemistry 2000;55:589-95.

8. Sereno D, Holzmuller P, Lemesre JL. Efficacy of second line drugs on antimony resistant amastigotes of Leishmania infantum. Acta Trop 2000;74:25-31.

9. Jha TK, Sundar S, Thakur CP, Bachmann P, Karbwang J, Fischer $\mathbf{C}$ et al. Miltefosine, an oral agent, for the treatment of Indian visceral leishmaniasis. N Engl J Med 1999;341:1795-800.

10. Perez-Victoria FJ, Castanys S, Gamarro F. Leishmania donovani resistance to Miltefosine involves a defective inward translocation of the drug. Antimicrob Agents Chemother 2003;47:2397-403.

11. Rodriguez N, Bailey BN, Martin MB, Oldfield E, Urbina JA, Docampo R. Radical cure of experimental cutaneous leishmaniasis by the bisphosphonate pamidronate. J Infect Dis 2002;186:138-40.

12. Henriquez W, Crescente O, Arrieche D, Marchan E. Biological activities of a kaurenic acid isolated from Wedelia trilobata. Nat Prod Res Mill 2002;2:38.

13. Coronado A. Propiedades biológicas y análisis fotoquímico de Wedelia trilobata y Tridax procumbens (Asteracea) (trabajo de Grado). Cumaná, Venezuela: Universidad de Oriente; 1998.

14. Ghisalberti EL. The biological activity of naturally occurring kaurane diterpenes. Fitotherapy 1997;68:30325.

15. Campos-Bedolla $\mathbf{P}$, Campos $\mathbf{M}$, Valencia-Sánchez $A$, Ponce-Montero $\mathrm{H}$, Uribe $\mathrm{C}$, Osuna $\mathrm{L}$ et al. Inhibitory action of kaurenoic acid derivatives from Montana tormentosa (Asteracea) on acetylcholine, oxytocin and serotonin induced rat uterine contractions. Phytother Res 1997;11:11.

16. Tincusi BM, Jimenez IA, Bazzocchi IL, Moujir LM, Mamani ZA, Barroso JP et al. Antimicrobial terpenoids from the oleoresin of the Peruvian medicinal plant Copaifera paupera. Plant Med 2002;68:808-12.

17. Brun R, Jenni L. A new semi-defined medium for Trypanosoma brucei sp. Acta Trop 1997;34:21-33.

18. Sereno D, Lemesre JL. Axenically cultured amastigotes forms as an in vitro model for investigation of anti leishmanial agents. Antimicrob Agents Chemother 1997;41:972-6.

19. Rodriguez NM, Docampo R, Lu Hg HG, Scott DA. Overexpression of the Leishmania amazonensis $\mathrm{Ca}^{2+}$ ATPase gene Imaa1 enhances virulence. Cell Microbiol 2002; 4:117-26. 
20. Huber W, Koella JC. A comparison of three methods of stimulating $\mathrm{EC}_{50}$, in studies of drug resistance of malaria parasites. Acta Trop 1993;55:527-61.

21. Bankowski Z, Howard-Jones N. Biomedical Research Involving Animals: Proposed International Guiding Principles. Geneva: WHO; 1985.

22. Bankowski Z, Howard-Jones N. International Guiding Principles for Biomedical Research Involving Animals. Geneva: WHO; 1986.

23. Ephros $M$, Bitnun A, Shaked $P$, Waldman $E$, Zilberstein D. Stage specific activity of pentavalent antimony against Leishmania donovani axenic amastigotes. Antimicrob Agents Chemother 1999;43:278-82.

24. Luque F, Fernández-Ramos R, Entrala E, Rosales MJ, Navarro JA, Romero MA et al. In vitro evaluation of newly synthesized $(1,2,4)$ triazolo $\left(1,5^{\mathrm{a}}\right)$ pyrimidine derivatives against Trypanosoma cruzi, Leishmania donovani and Phytomonas staheli. Comp Biochem Physiol 2000;126:39-44.

25. Alves LV, do Canto-Cavalheiro MM, CysneFinkelstein L, Leon LL. In vitro antiproliferative effects of several diaryl derivatives on Leishmania spp. Biol Pharm Bull 2003;26:453-6.
26. Ferreira IC, Lonardoni MV, Machado GM, Leon LL, Gobbi Filho L, Pinto LH et al. Anti leishmanial activity of alkaloidal extract from Aspidosperma ramiflorum. Mem Inst Oswaldo Cruz 2004;99:325-7.

27. Akendengue B, Ngou-Milama E, Laurens A, Hocquemiller R. Recent advances in the fight against leishmaniasis with natural products. Parasite 1999;6:3-8.

28. Tan N, Kaloga M, Radke O, Kiderin A, Oksiiz S, Ulubelen A et al. Abietane diterpenoids and triterpenoic acids from Salvia silicica and their anti leishmanial activities. Phytochemistry 2000;61:881-4.

29. Habtemariam S. In vitro anti-leishmanial effects of antibacterial diterpenes from two Ethiopian Premna species: $P$. shimperi and $P$. oligotricha. BMC Pharmacol 2003;3:6.

30. Germonprez N, Maes L, Van Puyvelde L, Van Tri $\mathbf{M}$, Tuan DA, De Kimpe N. In vitro and in vivo antileishmanial activity of triterpenoid saponins isolated from Maesa balansae and some chemical derivatives. J Med Chem 2005;48:32-7. 